\title{
Capacidade Funcional Máxima e Função Diastólica em Portadores de Cardiomiopatia Chagásica sem Insuficiência Cardíaca Congestiva
}

\author{
Charles Mady, Barbara M. Ianni, Edmundo A rteaga, Vera Maria Cury Salemi, Paulo R. Santos Silva, \\ Rita Helena A. Cardoso, Dalia Ballas
}

São Paulo, SP

Objetivo - Identificar disfunções cardíacas precoces em pacientes assintomáticos com cardiomiopatia chagásica.

Métodos - Foram estudados 38 indivíduos masculinos, sendo o grupo controle constituído de 20 indivíduos sedentários normais e o grupo Chagas de 18 pacientes assintomáticos, portadores da doença de Chagas, com eletrocardiograma alterado e fração de encurtamento (DD) normal ao ecocardiograma. Ambos os grupos foram submetidos à avaliação da capacidade funcional máxima, com medidas do consumo máximo de oxigênio ( $\left.\mathrm{VO}_{2} \max \right)$, índice de pulso de oxigênio ( $P \mathrm{O}_{2}$ max), ventilação máxima (VE max), freqüência cardíaca máxima (FC max), e limiar anaeróbio do $\mathrm{VO}_{2} \max \left(\mathrm{LA}-\mathrm{VO}_{2}\right)$. A função diastólica do ventrículo esquerdo foi avaliada pelo ecocardiograma convencional (ondas E e A além da relação E/A).

Resultados - Não ocorreram diferenças significativas entre os dois grupos em relação ao $D D(p=0,212)$ e a idade média $(p=0,060)$. Houve diferença significativa ( $p<0,001)$ em relação aos parâmetros $\mathrm{VO}_{2}$ max, $\mathrm{PO}_{2}$ max, $V E \max , F C \max , L A-V O_{2}$, onda E e relação E/A. Não houve significância $(p=0,520)$ em relação a onda $A$.

Conclusão - O comprometimento na função ventricular pode contribuir para as diferenças acima mencionadas, como conseqüência de disfunção sistólica e diastólica.

Palavras-chave: doença de Chagas, capacidade funcional máxima, função diastólica

\section{Maximal Functional Capacity and Diastolic Function in Patients with Cardiomyopathy due to Chagas' Disease without Congestive Heart Failure}

Purpose - To identify early cardiac dysfunctions in asymptomatic patients with Chagas' cardiomyopathy.

Methods - We studied 38 male individuals: control group consisting of 20 sedentary normal individuals and Chagas' group, of 18 asymptomatic patients with Chagas' disease, with suggestive electrocardiographic alterations and normal fractional shortening (FS) on echocardiogram. Both groups were submitted to evaluation of the maximal functional capacity, with measurements of maximal $\mathrm{O}_{2}$ consumption ( $\mathrm{VO}_{2}$ max), $\mathrm{O}_{2}$-pulse rate $\left(\mathrm{PO}_{2}\right.$ max), maximal ventilation (VEmax), maximal heart rate (HRmax), and anaerobic threshold of the $\mathrm{VO}_{2} \max \left(\mathrm{VO}_{2}-\right.$ AT). Left ventricular diastolic function was evaluated by conventional echocardiography ( $E$ wave, A wave and E/A ratio).

Results - No significant differences occurred between the two groups with regard to $F S(p=0.212)$ and age means $(p=0.060)$. The 2 groups were significantly different $(p<0.001)$ regarding the parameters $\mathrm{VO}_{2}$ max, $\mathrm{PO}_{2}$ max, VEmax, HRmax and $\mathrm{VO}_{2}-\mathrm{AT}$, E wave and E/A ratio. There was no difference $(p=0.520)$ in A wave.

Conclusion - Impaired ventricular function may account for the above mentioned differences, a consequence of both systolic and diastolic dysfunctions.

Key-words: Chagas' disease, maximal functional capacity, diastolic function

Arq Bras Cardiol, volume 69 (nº 4), 237-241, 1997

Instituto do Coração do Hospital das Clínicas - FMUSP

Correspondência: Charles Mady - Incor - Cardiopatias Gerais - Av. Dr. Enéas C. Aguiar, 44 - 05403-000 - São Paulo, SP

Recebido para publicação em 19/3/97

Aceito em 29/7/97
Desde a descrição da doença de Chagas ${ }^{1}$, nosso conhecimento de suas características tem melhorado em muitos aspectos. O envolvimento cardíaco, determinante fundamental da história natural da doença, tem sido estudado exaustivamente do ponto de vista morfológico, etiopa- 
togênico e clínico ${ }^{2-9}$. No entanto, estudos das alterações funcionais, hemodinâmicas e metabólicas são geralmente restritos a pacientes com insuficiência cardíaca, geralmente em estágios avançados ${ }^{9-11}$. A forma subclínica, que inclui a forma indeterminada com eletrocardiograma(ECG) normale um grupo de pacientes com ECG alterado, mereceu pouca atenção, particularmente com respeito a seus aspectos funcionais ${ }^{12-16}$. O grupo com ECG alterado pode ou não apresentar dilatação ventricular.

Pouco se conhece sobre a capacidade funcional dos pacientes com cardiomiopatia chagásica sem dilatação ventricular e insuficiência cardíaca. São pacientes que geralmente têm exame físico e atividade profissional normais. Portanto, é muito importante identificar possíveis disfunções cardíacas entre eles, as quais implicam na necessidade de seguimento clínico cuidadoso e possíveis intervenções terapêuticas precoces.

O objetivo deste estudo é identificar disfunções cardíacas precoces em pacientes com cardiomiopatia chagásica assintomática.

\section{Métodos}

Foram estudados dois grupos de indivíduos do sexo masculino: o grupo controle formado de 20 indivíduos sedentários normais com idades variando de 20 a 42 anos $(32,9 \pm 6,1)$, selecionados no mesmo ambulatório dos pacientes, e o grupo Chagas, consistindo de 18 pacientes com cardiomiopatia chagásica sem sintomas ou sinais clínicos de insuficiência cardíaca, com idades variando de 19 a 40 anos $(29,2 \pm 5,6)$ com reações sorológicas positivas para a doença de Chagas (Guerreiro-Machado e imunofluorescência) e alterações eletrocardiográficas sugestivas da doença. Estas foram constituídas por bloqueio de ramo direito (BRD) associado a bloqueio divisional ântero-superior esquerdo em seis casos, quatro com BRD, três com alterações difusas da repolarização ventricular (ADRV), dois com bloqueio atrioventricular de $1^{\circ}$ grau e ADRV, três com ADRV e extras-sístoles ventriculares. A função sistólica global e segmentar do ventrículo esquerdo (VE), analisada pelo ecocardiograma, foi normal ao repouso. Todos os pacientes foram acompanhados, ambulatorialmente e não fizeram uso de medicação.

O estudo ecocardiográfico ao repouso foi obtido no aparelho Aloka SSD 870. Para avaliar a função sistólica do VE foram analisadas a média de cinco batimentos consecutivos do diâmetro diastólico final (DDF), diâmetro sistólico final (DSF), em centímetros (cm) e a fração de encurtamento (DD) do VE em $\%{ }^{17}$. A curva da velocidade do fluxo mitral foi obtida na ponta dos folhetos da valva mitral por onda de Doppler pulsado convencional ${ }^{18}$. A avaliação da capacidade funcional foi realizada utilizando o aparelho Beckman, mod MMC, munido de sensor polarográfico (OM-11)e sistema infravermelho (LB-2) para avaliação da fração expirada do oxigênio e do dióxido de carbono. O MMC foi calibrado previamente a cada exame com conhecimento anterior da amostra de gases. As variações respiratórias foram obtidas sob condições padronizadas de temperatura, pressão e umidade (StPD), utilizando-se fatores de correção apropriados. Os grupos foram submetidos a teste de esforço máximo emuma esteira monitorizada (Quinton, mod 18-54) com velocidade variáveis (mph) e inclinação (\%), usando o protocolo de Naughton modificado. Após 2 min na posição ereta,

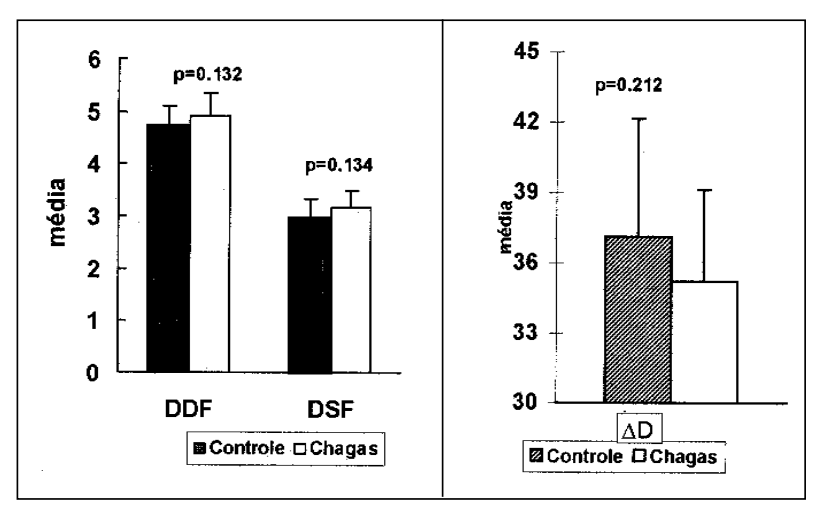

Fig. 1 - Representação gráfica dos resultados da análise comparativa dos diâmetros diastólico final (DDF) e sistólico final (DSF), e da fração de encurtamento (DD) entre os grupos controle e Chagas. As barras representam médias e as linhas verticais os desvios padrão.

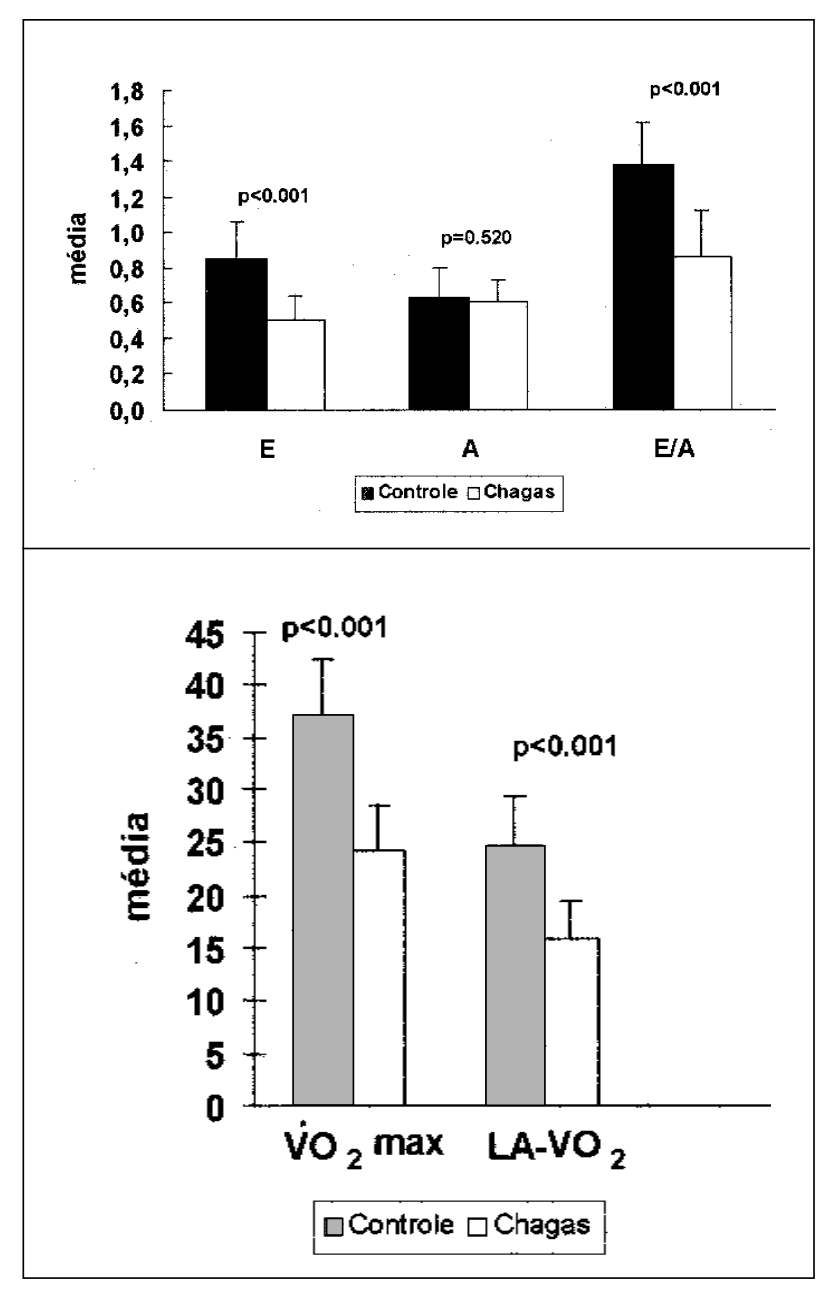

Fig. 2 - Representação gráfica dos resultados da análise comparativa dos parâmetros ecocardiográficos diastólicos: onda E, onda A e relação E/A entre os grupos controle e Chagas. As barras representam médias e as linhas verticais desvios padrão. 
sem exercício, todos os pacientes foram encorajados a realizar exercício progressivo, até que os sintomas de fadiga ou dispnéia os tornasse inábeis para continuar o exame. Em todos os pacientes foram obtidos os valores de consumo máximo de oxigênio $\left(\mathrm{VO}_{2} \max \right) \mathrm{em} \mathrm{ml} \cdot \mathrm{kg}^{-1} \cdot \mathrm{min}^{-1}$, o pulso de oxigênio $\left(\mathrm{PO}_{2} \max \right) \mathrm{em} \mathrm{ml/bat,} \mathrm{a} \mathrm{ventilação} \mathrm{máxima(VEmáx)}$ em 1/min, a freqüência cardíaca máxima (FC máx) em bpm, e limiar anaeróbio do $\mathrm{VO}_{2} \max \left(\mathrm{LA}-\mathrm{VO}_{2}\right) \mathrm{em} \mathrm{ml} \cdot \mathrm{kg}^{-1} \cdot \mathrm{min}^{-1}$. O limiar anaeróbio foi determinado de acordo com o aumento não-linear na ventilação, aumento abrupto no quociente respiratório, e aumento na produção de dióxido de carbono ${ }^{19}$.

Todos os pacientes concordaram em participar do estudo, após a explicação do objetivo dos exames.

A medida das variáveis dos grupos foram comparadas pelo teste t de Student para variáveis independentes. Foi considerado $\mathrm{p}<0,05$ como estatisticamente significativo. Todas as análises estatísticas foram realizadas com o programa SAS (Statistical Analysis System).

\section{Resultados}

As idades média do grupo controle $(32,9 \pm 6,1)$ e do grupo Chagas $(29,2 \pm 5,6)$ não foram estatisticamente significantes $(\mathrm{p}=0,060)$.

Os parâmetros ecocardiográficos $\operatorname{DDF}(4,72 \pm 0,38 \mathrm{~cm}$ e $4,90 \pm 0,35 \mathrm{~cm})$, DSF $(2,97 \pm 0,44 \mathrm{~cm} \mathrm{e} 3,17 \pm 0,33 \mathrm{~cm})$ e DD $(37,10 \pm 5,05 \%$ e $35,22 \pm 3,92 \%)$ não diferiram significativamente $(\mathrm{p}=0,132 ; 0,134 \mathrm{e} 0,212$, respectivamente $)$ nos grupos controle e Chagas, respectivamente (fig. 1).

Os parâmetros ecocardiográficos como onda $\mathrm{E}(0,85 \pm$ $0,21 \mathrm{~m} / \mathrm{s}$ e $0,50 \pm 0,14 \mathrm{~m} / \mathrm{s})$ e relação E/A $(1,37 \pm 0,24$ e $0,86 \pm$ $0,26)$, diferiram significativamente $(\mathrm{p}<0,001)$ nos grupos controle e Chagas, respectivamente. A variável onda A não diferiu significativamente ( $\mathrm{p}=0,520)$ entre os grupos (fig. 2).

As variáveis $\mathrm{VO}_{2} \max \left(36,98 \pm 5,45 \mathrm{ml} . \mathrm{kg}^{-1} \cdot \mathrm{min}^{-1} \mathrm{e} 24,32 \pm\right.$ $\left.4,25 \mathrm{ml} \cdot \mathrm{kg}^{-1} \cdot \mathrm{min}^{-1}\right), \mathrm{LA}-\mathrm{VO}_{2}\left(24,58 \pm 4,71 \mathrm{ml} \cdot \mathrm{kg}^{-1} \cdot \mathrm{min}^{-1} \mathrm{e}\right.$ $\left.15,81 \pm 3,65 \mathrm{ml} \cdot \mathrm{kg}^{-1} \cdot \min ^{-1}\right)$, VEmáx $(112,97 \pm 17,611 / \mathrm{mine}$ $50,13 \pm 13,491 / \mathrm{min})$, FC $\max (185,65 \pm 7,40 \mathrm{bpm}$ e $154,33 \pm$ 21,35bpm), ePO $\max (15,06 \pm 2,49 \mathrm{ml} / \mathrm{bate} 10,47 \pm 1,44 \mathrm{ml} / \mathrm{bat})$, tiveram diferença estatisticamente significativa $(\mathrm{p}<0,001)$ nos grupos controle e Chagas, respectivamente (fig. 3).

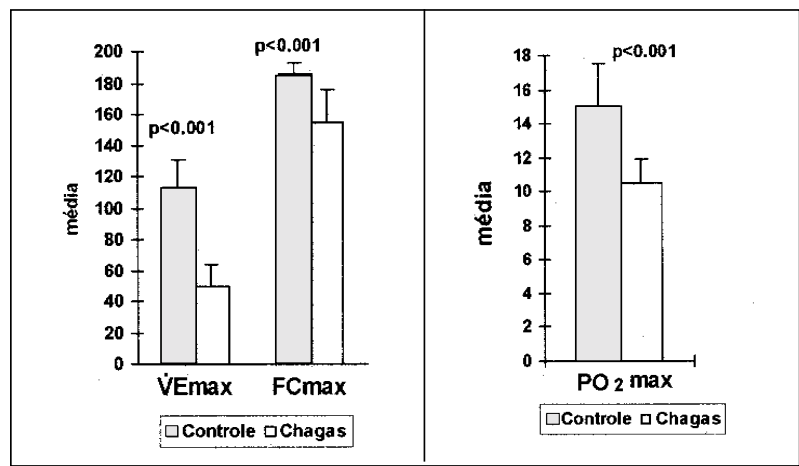

Fig. 3 - Representação gráfica dos resultados das análises comparativas do consumo máximo de oxigênio $\left(\mathrm{VO}_{2} \max \right)$, limiar anaeróbio do $\mathrm{VO}_{2} \max \left(\mathrm{LA}-\mathrm{VO}_{2}\right)$, ventilação máxima(VEmáx), frequiência cardíaca máxima(FCmáx), e pulsode oxigênio(POmáx), nos grupos controle e Chagas.

\section{Discussão}

A doença de Chagas é uma causa freqüente de cardiomiopatia dilatada. Sua apresentação clínica é caracterizada por graus variados de insuficiência cardíaca, com a mortalidade aumentando proporcionalmente com a elevação da classe funcional. A piora do prognóstico caminha em paralelo com a redução da fração de ejeção em repouso ${ }^{9}$. Infilltrado celular mononuclear intersticial associado a degeneração e fibrose nas biópsias endomiocárdicas obtidas do ventrículo direito (VD) foram observados em estudos prévios e representam as alterações patológicas básicas ${ }^{6,7}$. A quantidade de colágeno intersticial aumenta em proporção ao envolvimento cardíaco ${ }^{8}$.

No entanto, indivíduos com reações sorológicas positivas e alterações eletrocardiográficas sugestivas da doença de Chagas, mas sem sinais ou sintomas de insuficiência cardíaca são freqüentemente encontrados na prática clínica. Eles têm atividade física e profissional normal, pois são assintomáticos, e não são avaliados periodicamente. A despeito de estarem assintomáticos, as alterações eletrocardiográficas sugerem a presença de envolvimento miocárdico, que é confirmada por alterações detectadas nos fragmentos obtidos através de biópsia endomiocárdica do $\mathrm{VD}^{7,8}$. Muitos deles têm alta dos serviços médicos, e apenas retornam após longo tempo, quando a doença já avançou. Isto obviamente constitui um problema epidemiológico considerável.

Então, é importante caracterizar o estágio evolutivo desta doença, para fazer o melhor seguimento e para levantar a possibilidade de tratamento precoce da disfunção cardíaca.

O envolvimento ventricular na doença de Chagas é tradicionalmente conhecido por causar cardiomiopatia dilatada e dano na função sistólica. Estudos ecocardiográficos anteriores da doença cardíaca assintomática com alterações eletrocardiográficas mostraram pacientes com contratilidade normal ou alterada ${ }^{20}$. Alguns autores encontraram disfunção diastólica significativa nestes grupos ${ }^{21-23}$. Estes achados levaram os autores a acreditar que as manifestações iniciais da cardiomiopatia chagásica são predominantemente secundárias a disfunção diastólica em vez de sistólica. No presente estudo, foram analisados apenas pacientes com diâmetros e DD do VE normais ao repouso, estatisticamente semelhantes ao grupo controle.

Os pacientes chagásicos apresentaram valores menores da onda E e da relação E/A quando comparados com o grupo controle, sugerindo que eles apresentam uma disfunção diastólica, como foi descrito previamente ${ }^{21-23}$.

Como o grupo Chagas têm diâmetros e DD normais e padrão de relaxamento anormal em repouso, foi tentado encontrar alterações funcionais durante o exercício, denotando comprometimento da reserva cardíaca.

Apenas indivíduos do sexo masculino foram incluídos porque a capacidade funcional máxima é maior neste grupo quando comparado com pacientes do sexo feminino ${ }^{24}$. Eles foram submetidos a teste ergoespirométrico para avaliação 
do consumo máximo de oxigênio, que é uma medida objetiva e não invasiva da função cardíaca, de acordo com o princípio deFick. Foram obtidos o $\mathrm{PO}_{2}$ max, VEmáx, FCeLA-VO . Baseado no $\mathrm{PO}_{2} \max$, foi calculada a quantidade de oxigênio consumido em um ciclo cardíaco completo, que depende do volume sistólico e da diferença arteriovenosa de $\mathrm{O}_{2}$. Quanto maior é o $\mathrm{PO}_{2}$, maior é o volume de ejeção e o gradiente arteriovenoso de $\mathrm{O}_{2}$, até que o ponto máximo $\left(\mathrm{PO}_{2} \max \right)$ seja atingido. Isto demonstra a quantidade de $\mathrm{O}_{2}$ consumido do volume sangüíneo ejetado durante cada batimento cardíaco. $\mathrm{OVO}_{2}$ max e o $\mathrm{PO}_{2}$ max foram menores no grupo Chagas comparado com o controle e essas diferenças foram estatisticamente significativas, e refletem uma redução da reserva cardíaca no grupo Chagas. Em outras palavras, a disfunção cardíaca pode estar presente em um grau que não é suficiente para produzir sintomas ou alterar a DD. Esta, quando normal, não significa função sistólica normal, pois foi obtida em repouso. Como é sabido, alguns desses pacientes progridem para a forma dilatada da cardiopatia chagásica, a qual é caracterizada por disfunção sistólica predominante. Pacientes do grupo Chagas tem uma forma inicial de disfunção com valores menores de $\mathrm{VO}_{2}$ max e $\mathrm{PO}_{2}$ max secundários à associação de disfunção sistólica e diastólica.

Em estudos anteriores da capacidade funcional em pacientes com doença de Chagas em diferentes estágios ${ }^{25}$, concluímos que quanto menor a capacidade funcional máxima, maior é o dano cardíaco. No entanto, pacientes sem insuficiência cardíaca não foram estudados pelo ecocardiograma, com objetivo de caracterizar se dilatações das câmaras ventriculares estavam ou não presentes.

Pacientes com doença de Chagas têm redução da capacidade ventilatória quando comparados aos controles, como pode ser concluído através de valores menores do VE máx no grupo Chagas. A diminuição do débito cardíaco e a redução secundária do consumo máximo de $\mathrm{O}_{2}$ podem contribuir para uma redução da resistência dos músculos respiratórios durante o esforço máximo ${ }^{26}$.

A disfunção ventricular é conhecida por reduzir a capacidade do coração de aumentar a FC em resposta à diferentes estímulos. Em estudo realizado há 22 anos foi observada diminuição da resposta cronotrópica na doença de Chagas devido a alterações do sistema nervoso autônomo ${ }^{27}$.
Os valores menores da FC no grupo Chagas comparado com controle neste estudo demonstram que a doença de Chagas leva a uma redução na reserva cronotrópica.

O limiar anaeróbio têm características interessantes na doença de Chagas. É definido como a mais alta taxa metabólica na qual a energia é gerada somente do consumo de $\mathrm{O}_{2} \mathrm{e}$, portanto, sem anaerobiose concomitante. Comparando o LA- $\mathrm{VO}_{2}$ em ambos os grupos, observamos valores significativamente mais altos no grupo controle comparado ao Chagas, como seria esperado.

Nos estudos anteriores de fragmentos endomiocárdicos do VD de pacientes com doença de Chagas assintomática foram encontradas as mesmas alterações histológicas vistas em pacientes com insuficiência cardíaca ${ }^{7,8}$. Isto demonstra que a cardiomiopatia já está estabelecida a despeito da ausência de sintomas.

Portanto, estaé uma população de pacientes com doença miocárdica morfológica estabelecida, comECGe capacidade funcional máxima alterados e disfunção diastólica que permanecem assintomáticos e não mostram alterações na função sistólica ventricular esquerda em repouso. Quando submetidos ao exercício, estes pacientes mostram capacidade funcional máxima alterada, que pode ser explicada por redução da função ventricular esquerda sistólica e diastólica.

Estes pacientes têm doença cardíaca dilatada em potencial, a qual é silenciosa ao repouso. Essas informações podem auxiliar no diagnóstico precoce da cardiomiopatia dilatada e abrir a possibilidade para o tratamento precoce da insuficiência cardíaca. A evolução tardia responder-nos-á esta pergunta.

Concluindo, estes pacientes em fase inicial de cardiomiopatia chagásica têm alterações da velocidade de fluxo mitral e da capacidade funcional máxima, apesar de serem assintomáticos e de não terem dilatação de câmaras cardíacas à ecocardiografia convencional, fatos que demonstram a presença de disfunção cardíaca nesta fase evolutiva da doença. Assim, as alterações eletrocardiográficas observadas indicam comprometimento ventricular em fase inicial, fazendo-nos acreditar que esse comprometimento seja conseqüente a disfunções tanto sistólicas quanto diastólicas, com redução gradual da reserva cardíaca e possível evolução para as fases dilatadas da doença.

\section{Referências}

1. Chagas C - Nova tripanossomíase humana - Mem Inst Oswaldo Cruz 1909; 1 : 159-218.

2. Andrade ZA - Patogenia da doença de Chagas. Novos aspectos. Arq Bras Cardiol 1982; 38: 255-60.

3. Laranja FS, Dias G, Nóbrega E, Miranda A - Chagas' disease. A clinical, epidemiological and pathologic study. Circulation 1956; 14: 1035-60.

4. Fernández EA, Barretto ACP, Mady C, Ianni BM, Bellotti G, Pileggi F - O eletrocardiograma em pacientes com reações sorológicas positivas para doença de Chagas: Estudo de 600 casos. Arq Bras Cardiol 1985; 44: 333-7.

5. Mady C, Barretto ACP, Moffa PJ et al-O vetocardiograma na forma indeterminada da doença de Chagas. Arq Bras Cardiol 1985; 44: 83-5.
6. Mady C, Pereira-Barretto AC, Ianni BM, Lopes EA, Pileggi F - Right ventricular endomyocardial biopsy in undetermined form of Chagas' disease. Angiology 1984; 35: 755-9.

7. Pereira-Barretto ACP, Mady C, Arteaga-Fernández E et al - Right ventricular endomyocardial biopsy in chronic Chagas' disease. Am Heart J 1986; 111:307-12.

8. Mady C, Saldiva PHN, Caldini EG et al - Interstitial myocardium fibrosis is significantly associated with the degree of functional impairment in Chagas' cardiomyopathy. Eur Heart J 1994; 15(suppl): 590.

9. Mady C, Cardoso RHA, Barretto AC, da Luz PL, Bellotti G, Pileggi F- Survival and predictors of survival in patients with congestive heart failure due to Chagas' disease. Circulation 1994; 90: 3098-3102. 
10. Bestetti RB, Dal-Bó CMR, Freitas OC, Teno LAC, Castilho OT, Oliveira JSM. Noninvasive predictors of mortality for patients with Chagas' heart disease: a multivariate stepwise logistic regression study. Cardology 1994; 84: 261-7.

11. Carrasco HA, Parada H, Guerrero L, Duque M, Durán D, Molina C-Prognostic implications of clinical, electrocardiographic and hemodynamic findings in chronic Chagas' disease. Intern J Cardiol 1994; 43: 27-38.

12. Fernández EA, Barretto ACP, Mady C et al - Correlação entre as alterações eletrovetocardiográficas e o desempenho ventricular esquerdo na miocardiopatia chagásica crônica. Arq Bras Cardiol 1983; 41: 189-93.

13. Del Nero Jr E, Diament J, Papaléo Netto M et al - Parâmetros sistólicos fonomecanográficos do ventrículo esquerdo na forma pré-clínica da doença de Chagas crônica. Rev Hosp Clín Fac Med S Paulo 1974; 29(supl.): 383-9.

14. Friedmann AA, Armelin E, Nelken JR,Zerbini CAF, Coimbra MA, Serro Azul LG - Estudo ecocardiográfico do desempenho ventricular em fase pré-clínica da doença de Chagas. Rev Hosp Clín Fac Med S Paulo 1980; 35: 165-70.

15. Mady C, Décourt LV - A forma indeterminada da doença de Chagas. Arq Bras Cardiol 1981; 36: 143-5.

16. Mady C, Moraes AV, Galiano N, Décourt LV - Estudo hemodinâmico na forma indeterminada da doença de Chagas. Arq Bras Cardiol 1982; 38: 271-5.

17. Henry WL, DeMaria A, Gramiak R et al - Report of the american society of echocardiography committee on nomenclature and standards in two-dimensional echocardiography. Circulation 1980; 62: 212-7.

18. Nishimura RA, Housmans PM, Hatle LK, Tajik AJ - Assessment of diastolic function of the heart: Background and current applications of Doppler echocardiography. Mayo Clin Proc 1989; 64: 71-81.

19. Wasserman K, Whipp BJ, Koyal SN, Beaver WL - Anaerobic threshold and respiratory gas exchange during exercise. J Appl Physiol 1973; 35: 236-43.

20. Acquatella H, Schiller NB, Puigbó JJ et al - M-mode and two-dimensional echocardiography in chronic Chagas' heart disease. A clinical and pathologic study. Circulation 1980; 62: 787-99.

21. Caeiro T, Amuchastegui LM, Moreyra E, Gibson DG - Abnormal left ventricular diastolic function in chronic Chagas' disease: an echocardiographic study. Intern J Cardiol 1985; 9: 417-24.

22. Combellas I, Puigbó JJ, Acquatella H, Tortoledo F, Gomez JR Echocardiographic features of impaired left ventricular diastolic function in Chagas' heart disease. Br Heart J 1985; 53: 298-309.

23. Sousa ACS, Marin-Neto JA, Maciel BC, Gallo Jr L, Amorim DS, Barreto-Martins LE - Disfunção sistólica e diastólica nas formas indeterminadas, digestiva e cardíaca crônica da moléstia de Chagas. Arq Bras Cardiol 1988; 50: 293-9.

24. Johnson RA, Palacios I - Dilated cardiomyopathies of the adult. N Engl J Med 1982; 307: 1051-8.

25. Mady C - Estudo da capacidade funcional máxima pela ergoespirometria em pacientes portadores da doença de Chagas. Arq Bras Cardiol 1986; 47: 201-5.

26. Mancini DM - Exercise limitations in congestive heart failure - I. Prog Cardiov Dis 1995; 37: 347-70.

27. Gallo Jr L, Marin-Neto JA, Manço JC, Rassi A, Amorim DS - Abnormal heart rate responses during exercise in patients with Chagas' disease. Cardiology 1975; 60: $147-62$. 